\title{
DYNAMIC STALL ANALYSIS OF S809 PITCHING AIRFOIL IN UNSTEADY FREE STREAM VELOCITY
}

\author{
H. R. Karbasian $\quad$ S. A. Moshizi $\quad$ M. J. Maghrebi * \\ Sun-Air Research Institute \\ Ferdowsi University of Mashhad \\ Mashhad, Iran
}

\begin{abstract}
In this paper, the dynamic stall of S809 airfoil that widely used in horizontal axis wind turbine, in different reduced frequencies is investigated. The simulation was carried out numerically handling NavierStokes equations. For this purpose, the segregated solver with SIMPLE algorithm was chosen to solve the momentum equations. The effect of turbulence on the flow field is taken into account using Shear Stress Transport (SST) $\kappa-\omega$ turbulence model. The obtained numerical results are compared with experimental and a few numerical results. The results indicate that the $\kappa-\omega$ SST model is in good agreement with experimental results for both steady and unsteady conditions. Furthermore, a nondimensional parameter, relating the acceleration of unsteady free stream velocity and acceleration of pitch motion (known as reduced frequency), is also investigated. In addition, the results show that any increase in the reduced frequency increases the instantaneous aerodynamic characteristics of oscillating airfoil.
\end{abstract}

Keywords: Dynamic stall, S809 airfoil, Reduced frequency, Aerodynamic characteristics.

\section{INTRODUCTION}

A famous phenomenon that occurs in horizontal-axis wind turbines (HAWT) is dynamic stall. In wind farms, the strong winds with altered direction and velocity magnitude cause dynamic stall. This fact results in dynamic blade loading and unexpected forces which may lead to some crucial problems, including the fatigue or undesirable rotor performance in power production. Therefore, considering this phenomenon is essential to prevent any unforeseen problems in design of rotor blades for HAWT. Many experimental and semi-empirical methods, such as Boddei-Leishman (BL), have been developed to assess the dynamic stall and predict the dynamic loads. Johansen [1], Gupta and Leishman [2], Gonzalez and Munduate [3] and Sheng et al. [4] are researchers who used BeddoesLeishman model to investigate the unsteady aerodynamics of S809 airfoil. Also, enormous numerical and experimental studies in dynamic stall issue have been carried out on the different types of airfoil during many years ago. An experimental deliberation was conducted to examine the dynamic stall characteristics of a NACA 23012 airfoil at Reynolds number of 1.5 million by Leishman [5]. Ramsay et al. [6] studied the effects of airfoil roughness in Ohio State University AARL subsonic wind tunnel under steady state and unsteady conditions. They studied the unsteady behavior with varying pitch oscillations at different mean angles of attack, frequencies and amplitudes for Reynolds num- bers of $0.75,1,1.25$, and 1.4 million numerically. Next, Somers [7] measured a two dimensional S809 aerodynamic coefficients in different conditions for a range of Reynolds numbers experimentally. Sadeghi et al. [8] investigated the unsteady wake of an oscillating EPPLER 361 airfoil in subsonic wind tunnel by hotwire anemometry by varying pitching amplitude and mean angle of attack. Wang et al. [9] probed unsteady flow around the NACA0012 airfoil at low Reynolds number $\left(\approx 10^{5}\right)$. In their simulations, $2 \mathrm{D}$ geometrical configurations were employed to model the experimental investigations and a 2D incompressible unsteady CFD solver based on the finite volume method, was also employed to solve the full Unsteady Reynolds Averaged Navier-Stoks (URANS) governing equations. The calculations have been carried out using the standard $\kappa-\omega$ model, assuming that the flow over the airfoil is fully turbulent and the $\kappa-\omega$ SST model with the prediction of the laminar-to-turbulence transitional process. It is found that URANS with advanced turbulence models, such as the $\kappa-\omega$ SST model, are useful for the fast design or research intension for low Reynolds number airfoils and vertical-axis wind turbine, because they are capable of capturing the experimental data in a significant part of the flow dynamics.

Amiralaei et al. [10] studied a 2-D flow around a NACA0012 airfoil performing dynamic pitching motion in low Reynolds number regime. In their study, CFD method was utilized to solve Navier-Stokes equations discretized based on the finite volume method.

\footnotetext{
Corresponding author (mjmaghrebi@um.ac.ir)
} 
They investigated the effects of some unsteady flows and system parameters such as amplitude of oscillation, reduced frequency $\left(k=\pi f_{c} / U_{\infty}\right)$ and Reynolds number $\left(U_{\infty} c / v\right)$ on the instantaneous force coefficients and flow patterns. Gharali and Johnson [11] numerically studied an oscillating freestream over a stationary S809 airfoil using ANSYS Fluent 12.1 for several Reynolds number in the wide range of range of reduced frequency between 0.026 and 18 . In their simulation, laminarturbulent transition was simulated with the realizable $\kappa-\varepsilon$ and $\kappa-\omega$ SST models. For simulation of unsteady flow, they altered the direction of the far-field flow over the stationary airfoil at each time step based on the sinusoidal equation to simulate a proper wind direction for the boundary conditions. They founded that the behavior of aerodynamic coefficients, vorticity fields and velocity fields are very sensitive to the reduced frequency. Increasing the reduced frequency changes the arrangement of the vortices and alters the wake velocity profile from momentum deficit to momentum excess resulting in thrust generation. Also, decreasing the Reynolds number does not change the overall shape of the wake velocity profile, but the wake velocity profile shows both wake and jet structures at high reduced frequencies. Recently, Lu et al. [12] numerically investigated various unsteady parameters to analyze the effects of asymmetric sinusoidal motion on pitching airfoil aerodynamics for 2-D flow around a NACA0012 airfoil at $\mathrm{Re}=1.35 \times 10^{5}$. In their study, the $\kappa-\omega \mathrm{SST}$ turbulence model with transition was employed for the simulation. They mentioned that the $\kappa-\omega$ SST turbulence model has shown a quite dissipative character that attenuates the instabilities and the vortex structures related to the dynamic stall. They showed that the asymmetric has noticeable effects on the aerodynamic performance, as it affects the instantaneous force coefficient, maximum lift and drag coefficient, hysteresis loops and the flow structures.

As mentioned above, many researchers tried to evaluate the effect of different kinematic parameters and flow characteristics on the dynamic stall phenomenon. They also considered effect of different conditions and unsteadiness on wake structures being created behind the airfoil. In this paper, the unsteady aerodynamic characteristics and dynamic stall of S809 airfoil is also numerically investigated using CFD approach. The URANS equations in association with $\kappa-\omega$ SST turbulence model are solved to simulate the flow over a 2D airfoil. At first, the obtained results are analyzed and compared with experimental data. Subsequently, the different aspects of unsteady conditions on oscillating S809 airfoil are also evaluated. The free stream is accelerated to evaluate the effect of unsteady flow on dynamic stall phenomenon and find out how the aerodynamic forces may change during this process.

\section{GOVERNING EQUATIONS}

Full conservative Navier-Stokes equations for twodimensional unsteady flow considering no body force are used to simulate the flow filed as follows

$$
\frac{\partial Q}{\partial t}+\frac{\partial F}{\partial x}+\frac{\partial G}{\partial y}=\frac{\partial F_{v}}{\partial x}+\frac{\partial G_{v}}{\partial y}
$$

where $Q$ is the conservative vector, $F$ and $G$ are inviscid flux vectors, and $F_{v}$ and $G_{v}$ are viscous flux vectors which may be written as

$$
\begin{gathered}
Q=\left[\begin{array}{c}
\rho \\
\rho u \\
\rho v \\
\rho e_{t}
\end{array}\right], \quad F=\left[\begin{array}{c}
\rho u \\
\rho u^{2}+P \\
\rho u v \\
\rho u h_{t}
\end{array}\right], G=\left[\begin{array}{c}
\rho v \\
\rho u v \\
\rho v^{2}+P \\
\rho v h_{t}
\end{array}\right], \\
F_{v}=\left[\begin{array}{c}
0 \\
\tau_{x x} \\
\tau_{x y} \\
u \tau_{x x}+v \tau_{x y}-q_{x}
\end{array}\right], G_{v}=\left[\begin{array}{c}
\tau_{y y} \\
u \tau_{x y}+v \tau_{y y}-q_{y}
\end{array}\right]
\end{gathered}
$$

with $\tau_{x x}, \tau_{x y}$, and $\tau_{y y}$ being stress tensor components and $q_{x}$ and $q_{y}$ conductive heat transfer in $x$ - and $y$-directions, respectively. For the turbulence closure, according to two-equation Shear Stress Transport (SST) turbulence model proposed by Menter [13], the combined model of the $\kappa-\omega$ model and the $\kappa-\varepsilon$ model may be written as

$$
\begin{gathered}
\partial(\rho \kappa) / \partial t+\partial\left(\rho u_{j} \kappa\right) / \partial x_{j}=\frac{\partial}{\partial x_{j}}\left[\left(\mu+\sigma_{k} \mu_{t}\right) \frac{\partial \kappa}{\partial x_{j}}\right]+P_{k} \\
-\beta^{*} \rho \omega \kappa\left[1+\alpha_{1} M_{t}^{2}\left(1-F_{1}\right)\right]+\left(1-F_{1}\right) \overline{p^{\prime \prime} d^{\prime \prime}} \\
\partial(\rho \omega) / \partial t+\partial\left(\rho u_{j} \omega\right) / \partial x_{j}=\frac{\partial}{\partial x_{j}}\left[\left(\mu+\sigma_{\omega} \mu_{t}\right) \frac{\partial \omega}{\partial x_{j}}\right] \\
+\alpha \frac{\omega}{\kappa} P_{k}+2\left(1-F_{1}\right) \frac{\rho \sigma_{\omega_{2}}}{\omega} \frac{\partial \kappa}{\partial x_{j}} \frac{\partial \omega}{\partial x_{j}} \\
-\beta \rho \omega^{2}+\left(1-F_{1}\right) \beta^{*} \alpha_{1} M_{t}^{2} \rho \omega^{2}-\frac{\rho}{\mu_{t}}\left(1-F_{1}\right) \overline{p^{\prime \prime} d^{\prime \prime}}
\end{gathered}
$$

with $P_{k}$ being the production of turbulence. Turbulent Mach number, $M_{t}=\sqrt{2 k / c^{2}}$, and pressure dilatation, $\overline{p^{\prime \prime} d^{\prime \prime}}=-\gamma_{2} P_{k} M_{t}^{2}+\gamma_{3} \rho \beta^{*} \kappa \omega M_{t}^{2}$, correct the compressibility effects of the turbulent compressible flow. The coefficients of transport equations, $\sigma_{k}, \sigma_{\varpi}, \alpha$ and $\beta$ are calculated via the blending function, $F=$ $\tanh \left\{\min \left[\max \left(\frac{\sqrt{\kappa}}{\beta^{*} \omega d}, \frac{500 \vartheta}{\omega d^{2}}\right), \frac{4 \rho \sigma_{\bar{\omega}_{2}} \kappa}{C D_{k \varpi} d^{2}}\right]\right\}$, using the following equation

$$
\varphi=F_{1} \varphi_{1}+\left(1-F_{1}\right) \varphi_{2}
$$

which $\varphi$ represents each of mentioned coefficients and subscripts 1 and 2 correspond to $\kappa-\omega$ and $\kappa-\varepsilon$ turbulence models, respectively. According to the SST model, the eddy viscosity, $\mu_{t}$, may be written as

$$
\mu_{t}=\frac{\alpha^{*} a_{1} \kappa}{\max \left[a_{1} \omega, F_{2}\right]}
$$


which constant $a_{1}$ is equal to 0.31 and coefficient $F_{2}$ is calculated from the following relation

$$
F_{2}=\tanh \left\{\left[\max \left(\frac{2 \sqrt{\kappa}}{\beta^{*} \omega d}, \frac{500 \vartheta}{\omega d^{2}}\right)\right]^{2}\right\}
$$

with $C D_{k \omega}=\max \left[2 \rho \sigma_{\omega_{2}} / \omega\left(\partial \kappa / \partial x_{j}\right)\left(\partial \omega / \partial x_{j}\right), \quad 10^{-10}\right]$ being the positive part of the cross diffusion term and $d$ is the distance to the nearest surface. Because the low-Reynolds effects only modify the near wall boundary layer, low-Re corrections of Wilcox [14] are applied only to the $\kappa-\omega$ part of the SST model for $a^{*}, a_{1}$ and $\beta^{*}$ coefficients.

\section{METHODOLOGY}

The unsteady flow field around the S809 airfoil is numerically simulated by employing 2D URANS equations. The simulation is carried out in ANSYS Fluent 12.1.15. The system of equations is treated with segregated solver and is discretized based on the finite volume method. Furthermore, the semi-implicit method for pressure linked equation (SIMPLE) algorithm is used for pressure-velocity coupling. For spatial discretization, the second-order upwind method is used in the momentum equation. The transient terms are also conducted using a second-order Euler implicit scheme. Additionally, the convergence criteria for residuals (continuity, momentum and turbulent parameters) are also. The number of iterations in each time step is also selected $10^{2}$ to ensure satisfactory results. The turbulence effect on flow field is accounted for using the $\kappa-\omega$ SST turbulence model. At the point of boundary conditions, for the entrance and discharge of fluid in computational domain the velocity inlet and pressure outlet conditions, respectively, are selected. Also, no-slip condition for airfoil surface is selected to set up the wall condition. A traditional simulation of the dynamic stall phenomenon has been sinusoidal pitching oscillation of an airfoil which is investigated in previous studies such as [11,15]. So, the oscillating freestream over a fixed airfoil signifies by,

$$
\alpha(t)=\alpha_{0}+\alpha_{a} \sin (\Omega t)
$$

where $\Omega=2 \pi f$ and $f$ is the oscillating frequency. Also, $\alpha_{0}$ and $\alpha_{a}$ are the mean angle between the wind and the relative velocity and the pitch oscillating amplitude, respectively. The oscillating frequency can also be defined as,

$$
f=\frac{U_{\infty} k}{\pi c}
$$

where $k$ is the reduced frequency. Moreover, in the case of unsteady flow the free stream velocity enhances linearly and plateaus in first periodic time, see Fig. 1. As it is shown in Fig. 1(a), the oscillating airfoil is subjected to free stream. The aifoil starts to oscillate with a certain angle of attack and rotational velocity (or reduced frequency). During oscillation, different forces

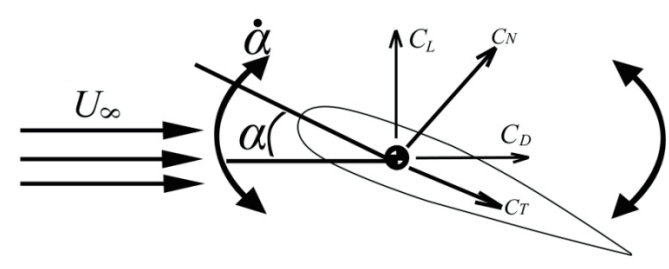

(a)

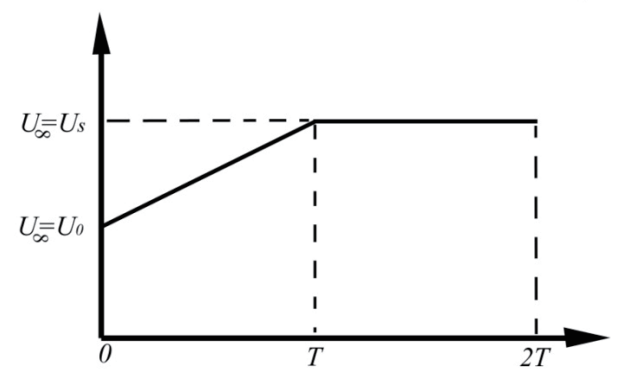

(b)

Fig. 1 Illustration of blade pitching motion and free stream velocity variations.

can be examined (lift, drag, normal and tangential) which will be discussed later. Figure 1(b) is also shows variation of free stream velocity. The stream velocity starts to increase until a certain time, and then it will be kept constant. Actually, oscillation of the airfoil shows the effect of wind direction variations on the airfoil and changes in free stream velocity demonstrates the wind velocity variations. However, the presented physical model shows the instantaneous effect of wind direction and velocity on airfoil which can be used in the analysis of the blades. It should be mentioned that all of simulation in this work is achieved based on the URANS with $\kappa-\omega$ SST turbulent model. Additionally, the considered Reynolds number, mean angle of attack, and pitch amplitude are $10^{6}, 8^{\circ}$ and $10^{\circ}$, respectively. It is worthy mentioning that the selected range of operating conditions is used in many previous literatures and investigations. Also, these ranges of parameters are also more applicable in wind turbine design, and this is why we selected these parameters and values.

\section{GRID GENERATION AND GRID INDEPENDENCY}

The accuracy of $\kappa-\omega$ SST turbulence model and the flow field are intensely related to the number of grid points close to the wall. The grid resolution for S809 airfoil must have some important features. First, the numerical accuracy of SST model is assured only for specific values of $y^{+}$of the nearest node to the blade surface. $y^{+}$is the non-dimensional wall distance for a turbulent boundary layer defined as $y^{+}=\rho u_{\tau} y / \mu$ and $u_{\tau}$ is the friction velocity. It has been reported in the literature that having a maximum value of $y^{+}$between 1 and 10 would give acceptable results $[16,17]$. Nevertheless, having a $y^{+}$lower than 1 leads to more accurate results. In the present study, the maximum value for 
$y^{+}$is equal to 3.5 which gives acceptable results while reducing the computational overhead (compared to the $y^{+}=1$ case) of the simulation $[18,19]$. First, the numerical accuracy of $\kappa-\omega$ SST model has to be reliable if $y^{+}$of the adjacent node to the blade surface would be lower than 5. On the other hand, better convergence of the solution is achieved when grid lines are perpendicular to the wall surface; consequently, boundary layer grid generation is used at the vicinity of the blade surface. In this type of mesh, the grid lines are normal to the surface near the wall. According to Fig. 2 the grid system was divided into three regions L1, L2 and L3 for fine and coarse meshes. The boundary of the computational domain L1 and L2 was located approximately $12.5 \mathrm{c}$ and $20 \mathrm{c}$ from the airfoil, respectively, where $\mathrm{c}$ is the chord of airfoil. In order to investigate the grid independent of the solution and its accuracy and conservative properties, three different meshes are studied as Table 1. For each grid, the lift coefficient is calculated for three angles of attack at $R e=10^{6}$. As evident from Table 2, there is a little difference between the numerical results of MI and MII meshes. Therefore, further results are obtained using the mesh of MII grid points. Figure 3 shows C-type grid generation around S809 airfoil. The grid generation of physical domain is performed using GAMBIT 2.3 software.

\section{RESULTS AND DISCUSSION}

\subsection{Validation Study}

In this section, the numerical results obtained are assessed in comparing with experimental and numerical results of Somers [7] and Johansen [1], respectively. Fig. 4 represents the aerodynamic coefficients at $R e=$ $10^{6}$ for the static case. It should be noted that the lift and drag coefficients are defined as $C_{L}=$

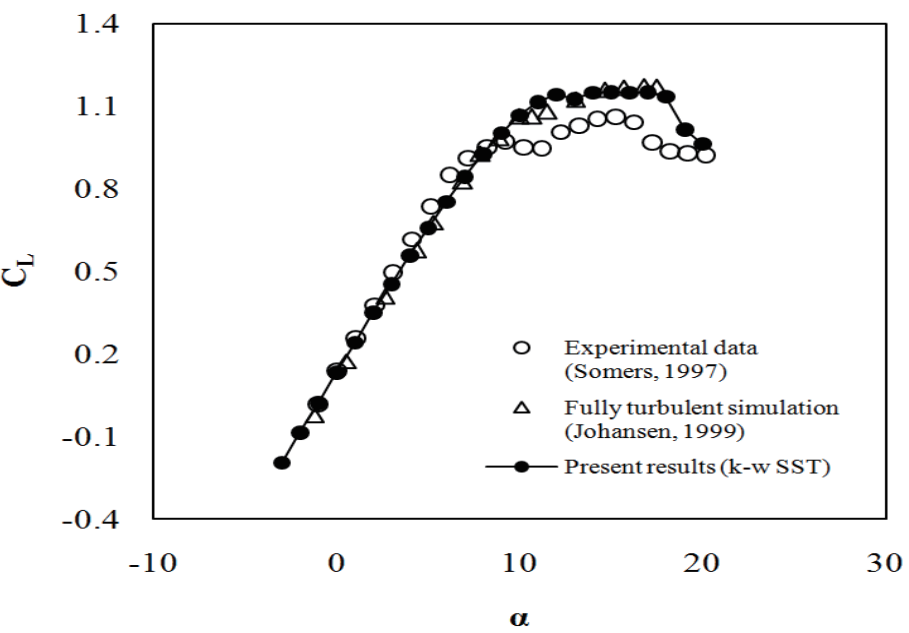

(a) lift
$L /\left(0.5 \rho U_{\infty}^{2} c\right)$ and $C_{D}=D /\left(0.5 \rho U_{\infty}^{2} c\right)$, respectively. Where $L$ and $D$ are lift and drag forces, respectively. The experimental results reported by Somers [7], illustrates the lift coefficient in Fig. 4(a) increases linearly with respect to angle of attack of $9^{\circ}$ and starts to decrease up to $11.2^{\circ}$ approximately. The lift coefficient

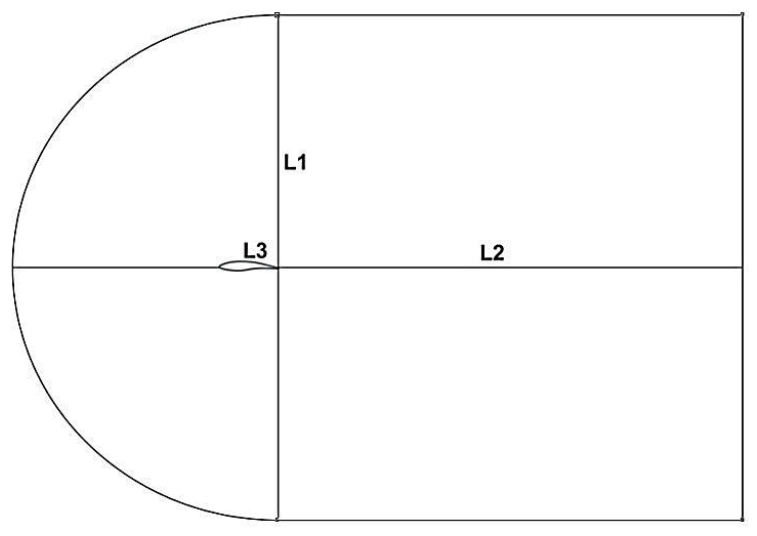

Fig. 2 Solution domain.

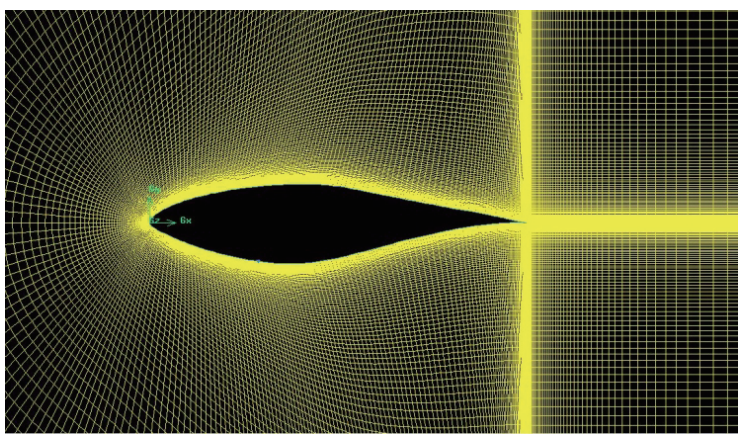

Fig. 3 C-type mesh around S809 airfoil.

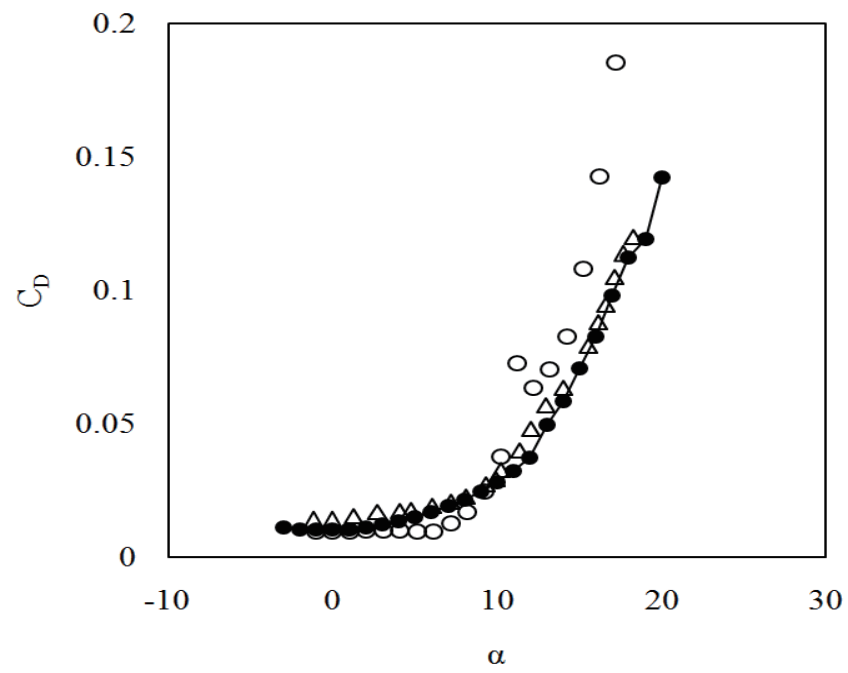

(b) drag

Fig. 4 Comparison of aerodynamic coefficients in static case. 
Table 1 Number of girds in three cases.

\begin{tabular}{|c|c|c|c|}
\hline & L1 & L2 & L3 \\
\hline MI & 40 & 40 & 80 \\
\hline MII & 60 & 50 & 100 \\
\hline MIII & 140 & 100 & 150 \\
\hline
\end{tabular}

Table 2 Comparison of aerodynamic coefficients for three cases with different grid sizes.

\begin{tabular}{|c|c|c|c|c|c|c|}
\hline \multirow{5}{*}{} & \multicolumn{6}{|c|}{ Angle of Attack $(\alpha)$} \\
\cline { 2 - 7 } & \multicolumn{2}{|c}{$0^{\circ}$} & \multicolumn{2}{c|}{$5^{\circ}$} & \multicolumn{2}{c|}{$10^{\circ}$} \\
\cline { 2 - 7 } & $C_{L}$ & $C_{D}$ & $C_{L}$ & $C_{D}$ & $C_{L}$ & $C_{D}$ \\
\hline MI & 0.25234 & 0.05234 & 1.02345 & 0.07244 & 1.312345 & 0.13451 \\
\hline MII & 0.13232 & 0.01991 & 0.65754 & 0.02468 & 1.067082 & 0.03645 \\
\hline MIII & 0.12724 & 0.02053 & 0.65657 & 0.02482 & 1.085 & 0.03674 \\
\hline
\end{tabular}

starts to fall in the stall region after angle of attack of $15^{\circ}$. Johansen [1] numerically modeled the flow over airfoil based on the fully turbulent simulation. Their lift coefficient results are in agreement with experimental one until $9^{\circ}$ and afterwards the results overestimate the lift coefficient up to $20^{\circ}$ roughly. The present lift coefficient based on the SST $\kappa-\omega$ model matches well with the experimental results for small angles of attack, and it follows the trend of lift coefficient results reported by Johansen properly. Figure 4(b) shows that the drag coefficient results are in good agreement with the experimental one for small angles of attack. By increasing the angle of attack, the numerical results will predict lower values than experimental investigations. Although at a higher angle of attack the present results may have a considerable difference with experimental results, it matches the numerical results reported by Johansen [1].
The unsteady aerodynamics of S809 airfoil at $R e=$ $10^{6}$ is depicted in Fig. 5 which shows aerodynamic coefficients for the oscillating S809 airfoil for $\alpha_{0}=8^{\circ}$ and $\alpha_{a}=10^{\circ}$. Furthermore, the reduced frequency is chosen 0.026 and the far field pressure is assumed to be atmospheric pressure. For validation of numerical modeling in dynamic case, the results are compared with reported results by other literatures. The experimental measurements for the same conditions are reported by Ramsay et al. [6]. Additionally, the Gupta and Leishman [2] used BL method to predict the unsteady aerodynamic coefficients at identical conditions. Furthermore, Gharali and Johnson [11] validated their numerical results for $\mathrm{S} 809$ airfoil with two other works. They used a Realized $\kappa-\varepsilon$ and $\kappa-\omega$ SST models in their study which are provided in the recent validation.

In Fig. 5(a) the instantaneous lift coefficients with respect to angle of attack are shown. The lift coefficient $C_{L}$ is approximately linear at low angles of attack up to $12^{\circ}$ and afterwards $C_{L}$ slope starts to decrease. The maximum $C_{L}$ occurs at the angle of attack of $15^{\circ}$ and decreases with an increase in angle of attack. When the pitching direction of airfoil is reversed, the angle of attack begins to decrease and this periodic motion (upstroke and downstroke) leads to a loop for aerodynamic characteristics. The instantaneous drag coefficient $C_{D}$ is also shown in Fig. 5(b). A rise in the angle of attack increases $C_{D}$, but the variations of $C_{D}$ at downstroke are not noticeable rather than those of lift for experimental and BL method results. As shown in Fig. 5, the present results predict the lift curve well, except at higher angle of attack in which the adverse pressure gradients cause the reverse and separated flow. Furthermore, the present instantaneous drag is overestimated while in separated flow regimes, this underestimates the experimental drag coefficient. In overall, the present results have better prediction than numerical results reported by Gharali and Johnson [11] and in most cases the present results match well the experimental results.

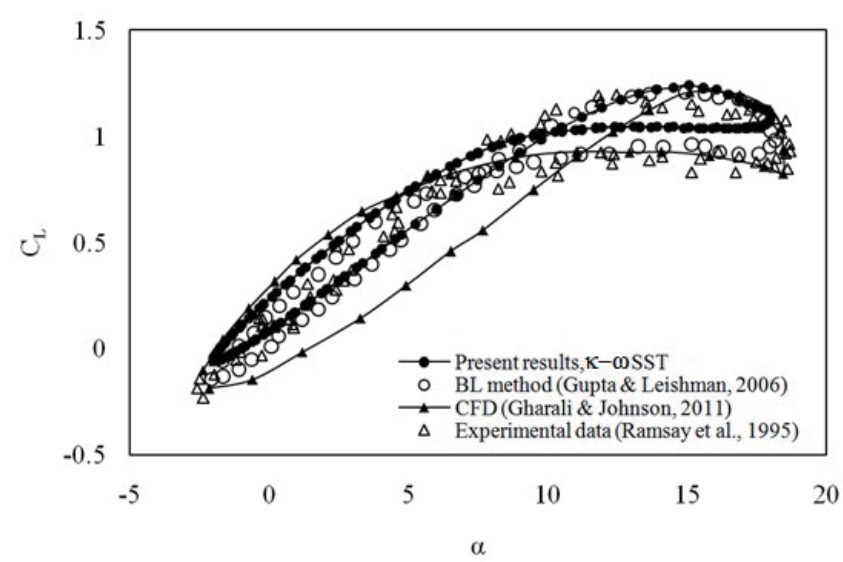

(a) lift

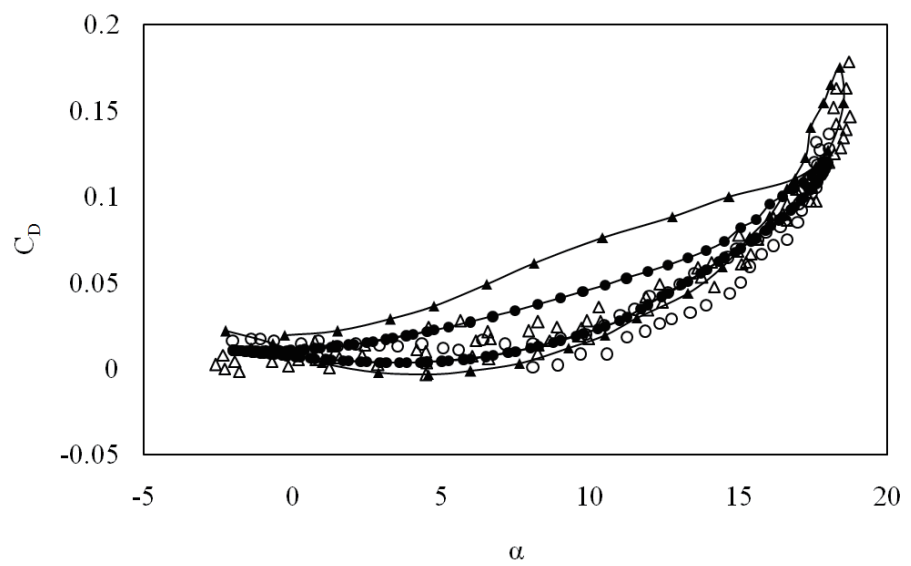

(b) drag

Fig. 5 Comparison of aerodynamic coefficients. 


\subsection{Effect of Reduced Frequency on Aerodynamics of Oscillating S809 Airfoil}

Figure 6 displays the unsteady aerodynamics of oscillating airfoil in different reduced frequencies. Figure 6(a) shows the variations of instantaneous force in $n$-direction with non dimensional time quantity. At $k=0.026$ the force begins to increase by increasing the angle of attack and then, dropped with some small fluctuations. This force drop occurs because the aerodynamic characteristics falls in stall region and its effect vanishes when the angle of attack decreases with time (downstroke). The negative angle of attack at 0.5 leads to negative force. At $k=0.1$ the produced force starts to increase with time (upstroke) and it decreases with angle of attack reduction (downstroke). As shown, a small force drop occurs at 0.2 without any fluctuations. This can also be demonstrated with decreasing in stall region. Additionally, the instantaneous force value is positive during the oscillating time. It should be noted that increasing the reduced frequency causes the stall phenomena to be postponed at higher angles of attack. At $k=0.5$ the force variations have a harmonic profile and stall region is postponed until higher angles of attack, which is not included in oscillating limits. At $k=0.5$ the maximum force reaches approximately 1.5 , which is the same for $k=0.25$. As observed by increasing the reduced frequency from

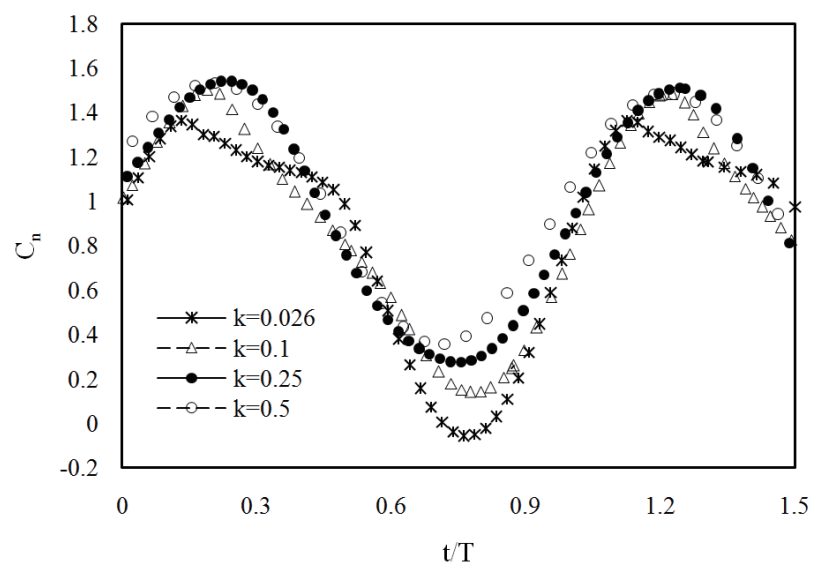

(a) $C_{n}$

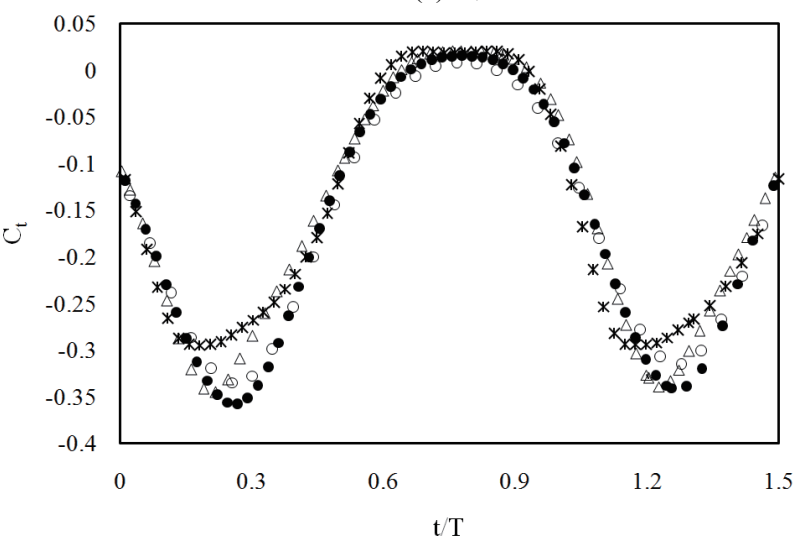

(b) $C_{t}$

Fig. 6 Instantaneous force coefficients in normal $(n)$ and tangential $(t)$ direction for oscillating airfoil in different reduced frequencies.
0.25 to higher values the stall region delays to higher angles of attack and increases the instantaneous force during oscillating. Figure 6(b) shows the instantaneous force in $t$-direction with respect to non-dimensional time. The negative value indicates the thrust in opposite direction of free stream velocity. If the airfoil is stationary, the force in $t$-direction is positive, because the only force in $t$-direction is drag. When the airfoil starts to oscillate, drag decreases and with any increase in the reduced frequency, this force reduces continuously. After a specified reduced frequency the drag switches to thrust. The reason is related to the reverse von-karman vortex produced behind the oscillating airfoil. Reversed vortex leads to a thrust production. As shown in Fig. 6(b), the stall occurs and the oscillating leads to thrust between the 0.026 and 0.1 . Furthermore, as reduced frequency increases, the maximum force in $t$-direction (thrust) is also increased. The overall conclusion which can be drawn is that the increase in reduced frequency could be postponed the stall phenomena and enhance the produced forces. Additionally, the airfoil may produce the positive force in $n$-direction even in the negative angle of attack.

The vortex contours of oscillating foil for $k=0.1$ are shown in Fig. 7. At $t=0.3$ the flow form trailing edge starts to separate and at $t=0.6$ the flow separation is moved toward leading edge. At $t=0.7$ the vortex is prepared for shedding and due to changes in angle of attack, at $t=0.8$ the vortex shedding happens. After $t=0.9$ the angle of attack decreases and flow separation moves toward trailing edge $(t=1.1,1.3$ and 1.5). At $t=1.7$ and 1.9 the flow attaches and the lift behavior is approximately linear. At $t=2.2$ the angle of attack starts to increase again and flow separation would occur at the trailing edge.

Figure 8 displays the contours of vortex for oscillating airfoil for $k=0.25$. As shown, the flow is separated from trailing edge and begins to move toward the leading edge. At $t=0.39$ the vortex starts to shed, but its size is smaller than the vortex in $k=0.1$. At $t=0.6$ the angle of attack reduces and separation point on upper surface of airfoil moves toward the trailing edge. At $t=1.04$ the angle of attack increases again and vortex formation will be completed at $t=1.1$ and 1.13 . From Fig. 7 and 8 it is concluded that the increase in reduced frequency causes the vortex size to be smaller and its distance to airfoil is closer. The vortex separation phenomenon caused by higher reduced frequency leads the airfoil to fall in dynamic stall later in comparing with cases with lower reduced frequencies. Additionally, the instantaneous lift coefficient in dynamic stall for the airfoil with higher frequencies is more pronounced than those of lower frequencies.

\subsection{Effect of Unsteady Free Stream Velocity on Aerodynamics of Oscillating Foil}

The unsteady condition consists of a uniform free stream and the oscillation of foil causes the variations of the effective angle of attack and as a result the aerodynamics behavior of airfoil. This unsteady condition 


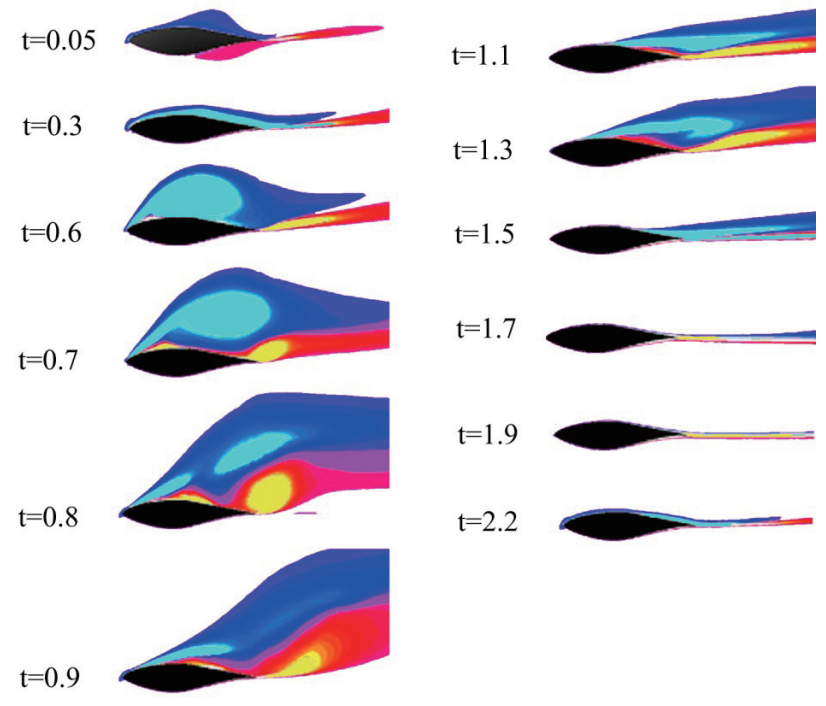

Fig. 7 Contours of vortex around oscillating airfoil at $k=0.1$ and $R e=10^{6}$.
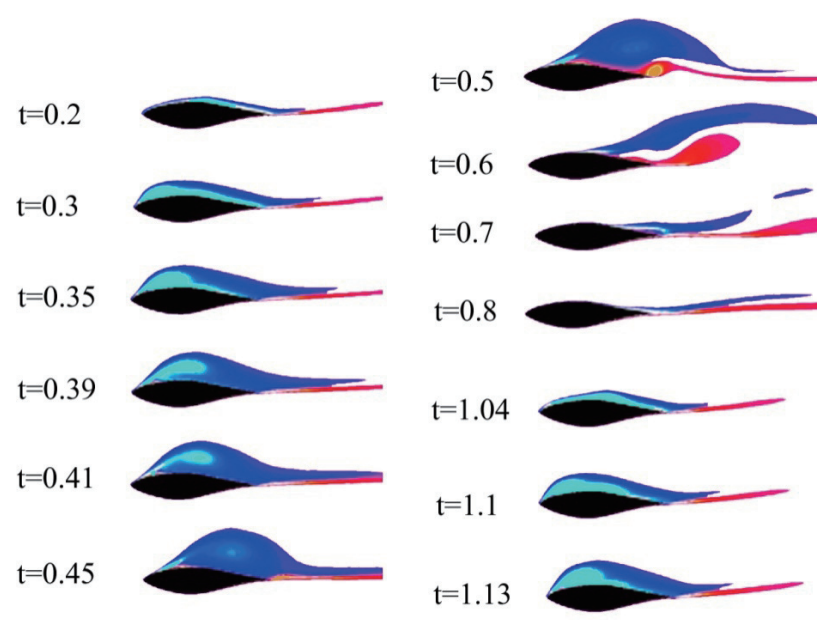

Fig. 8 Contours of vortex around oscillating airfoil at $k=0.25$ and $R e=10^{6}$.

is introduced using a well-known non-dimensional parameter, called as reduced frequency. The aim of this work is to consider the effect of unsteady free stream with contribution of airfoil oscillation. Thus, a nondimensional parameter is defined as follows:

$$
K^{\prime}=\frac{\frac{d U_{\infty}}{d t}}{\frac{d^{2} \alpha}{d t^{2}}}=\frac{\frac{d U_{\infty}}{d t}}{\alpha_{a} \Omega^{2}}
$$

This parameter introduces the acceleration of free stream velocity relative to the acceleration of rotational oscillation. The reduced frequency compares the velocity of oscillation with free stream velocity. This parameter is different with reduced frequency. The free stream velocity profile is chosen as linear function to have acceleration at inlet velocity in computational domain (see Fig. 1).

The aerodynamics of S809 airfoil in different $K^{\prime}$ is shown in Fig. 9. The instantaneous force in n- direction $C_{n}$ represents the effect of unsteady free stream
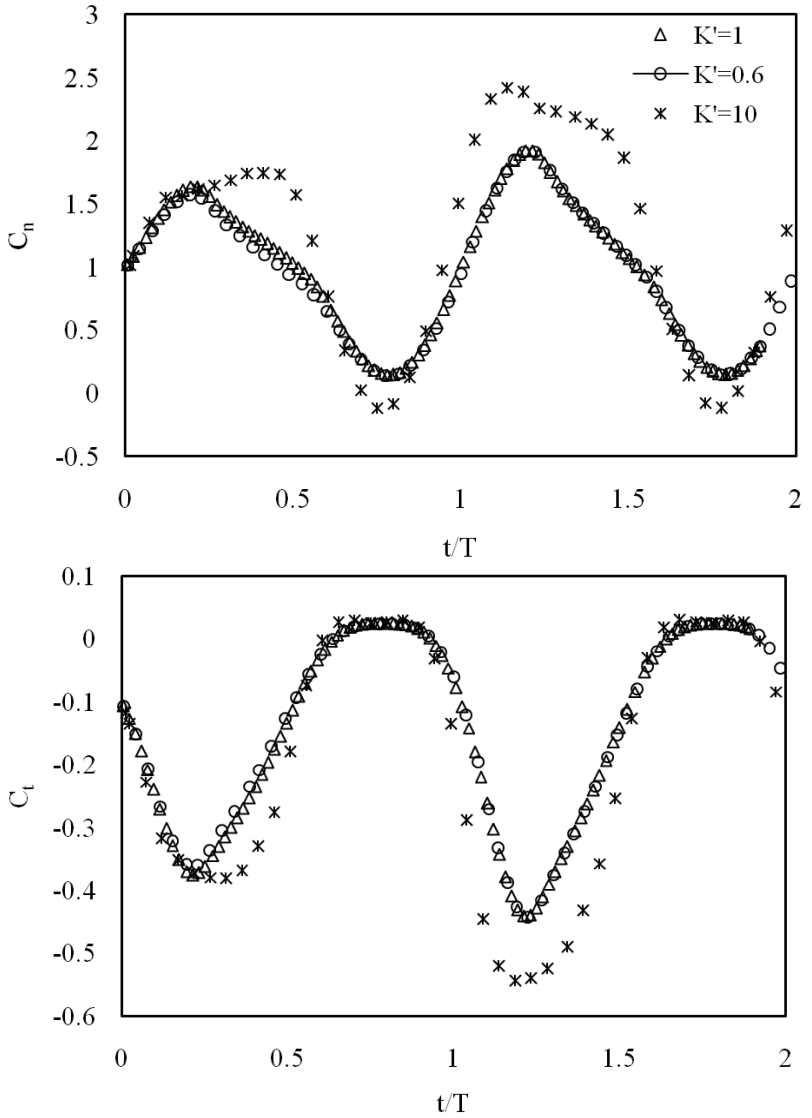

Fig. 9 Instantaneous forces in $n$ - and $t$-direction for oscillating airfoil with unsteady free stream velocity at different $K^{\prime}$.

velocity on aerodynamics (Fig. 9(a)). At $K^{\prime}=0.6$ the force begins to increase at upstroke and reduces at downstroke. As shown in Fig. 9(a), the second peak of $C_{n}$ is higher than the first one, because of the free stream velocity which increases continuously until $t / T=1$ and remains constant value afterwards. At $K^{\prime}=1, C_{n}$ follows the same trend between $t / T=0.2$ and $t / T=0.5$. This has higher value relative to that of $K^{\prime}=0.6$. After $t / T=0.6, C_{n}$ at $K^{\prime}=1$ coincides with that in $K^{\prime}=0.6$, because of the velocity variations vanished at $t / T=0.6$. At $K^{\prime}=10, C_{n}$ increases with increase in free stream velocity relative to previous $K^{\prime}$. At $t / T=0.15$ the airfoil begins to fall in stall region and its slope of lift coefficient curve is decreased. Figure 9(b) displays the instantaneous generated force coefficient in $t$-direction $C_{t}$ for different $K^{\prime}$. As indicated any increase in $K^{\prime}$ could increase thrust production. At $K^{\prime}=0.6$ and $K^{\prime}=1$ the produced thrust contains a negligible difference, but at $K^{\prime}=10$ the produced thrust increases significantly relative to those in small $K^{\prime}$. Therefore, to increase $K^{\prime}$, different procedure exists. It is possible to either increase the free stream velocity magnitude or increase the slope of free stream velocity variations, and even reduce the airfoil oscillation frequency.

As mentioned above, the dynamic stall is one of the important concepts that must be taken into account in the design of wind turbine blades. Since the wind speed changes continuously by magnitude and direction, 
the blades will be undergone to dynamic stall phenomenon. Since the aerodynamic forces play a notable role in the design of wind turbine blades, it is possible to forecast the amount of extracting power by the wind turbine. By considering the instantaneous aerodynamic forces in dynamic stall problems, it can be found the amount of maximum aerodynamic forces and their average values out to prevent any failure or structural problem. The most of dynamic stall investigations are based on the oscillation of the foil in constant free stream velocity. Here, we also accelerated the free stream velocity to see the effect of unsteady free stream velocity on the aerodynamic forces. Thus, this type of consideration is useful to evaluate wind turbine blades since the wind speed may change during oscillation of blades. As illustrated above, the variations of free stream velocity during oscillation of foil have notable effects on the aerodynamic forces and it may be considered further in the analysis of dynamic stall.

\section{CONCLUSIONS}

In this study, the unsteady aerodynamics of oscillating S809 airfoil is investigated. The airfoil results are numerically obtained by solving Navier-Stokes equations using $\kappa-\omega$ SST model. A non-dimensional parameter which relates the acceleration of unsteady free stream velocity and the acceleration of rotational motion (pitch motion) is also introduced. The results can be highlighted as follow:

- At specified angle of attack range any increase in reduced frequency postpones the dynamic stall which increases $C_{n}$ and decreases $C_{t}$.

- With an increase in reduced frequency, $C_{n}$ reaches a maximum value and later on the reduced frequency do not have any effects on $C_{n}$.

- After higher reduced frequencies, $C_{n}$ follows a harmonic sinusoidal behavior.

- In higher reduced frequencies, the instantaneous $C_{t}$ is positive thrust.

- Any increase in reduced frequency causes the decrease in size of vortices formed around the airfoil.

- When free stream velocity increases with a constant acceleration the aerodynamic characteristics decreases and asymptotically reaches a constant value.

- Increase in $K^{\prime}$ may lead to increase in the instantaneous $C_{n}$ and $C_{t}$.

Since the unsteady wind characteristics are crucial challenge in aerodynamics of blades these conclusions can be applied in design of HAWT. Furthermore, these results can be applied to understand the other feature of oscillating objects when the unsteady conditions are more pronounced.

\section{NOMENCLATURES}

$C_{D} \quad$ drag coefficient

$C_{L} \quad$ lift coefficient

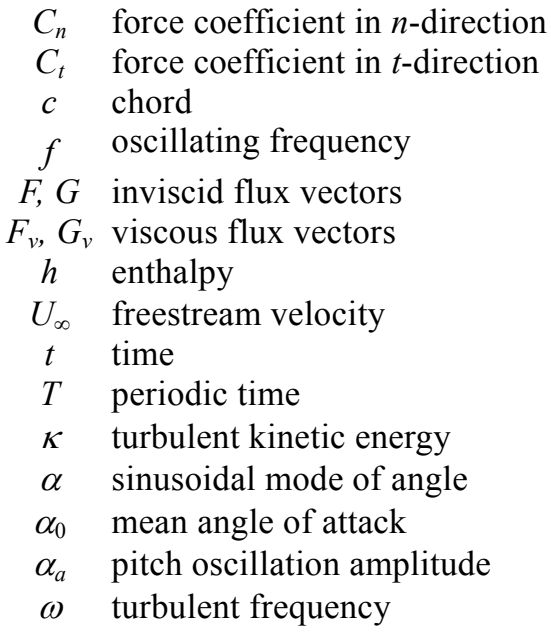

\section{ACKNOWLEDGEMENTS}

We thank our colleagues from Mechanical Engineering Department of Ferdowsi University of Mashhad who provided insight that greatly assisted the research, although they may not agree with all of the interpretations of this paper. We also express our gratitude toward our families for their kind co-operation and encouragement which help us in completion of this paper.

\section{REFERENCES}

1. Johansen, J., Unsteady Airfoil Flows with Application to Aeroelastic Stability, Risø National Laboratory, Roskilde, Denmark (1999).

2. Gupta, S. and Leishman, J. G., "Dynamic Stall Modelling of the S809 Aerofoil and Comparison with Experiments," Wind Energy, 9, pp. 521-547 (2006).

3. Gonzalez, A. and Munduate, X., "Unsteady Modelling of the Oscillating S809 Aerofoil and NREL Phase VI Parked Blade Using the BeddoesLeishman Dynamic Stall Model," Journal of Physics: Conference Series, 75, pp. 012020-012028 (2007).

4. Sheng, W., Galbraith, R. A. M. and Coton, F. N., "On the S809 Airfoil's Unsteady Aerodynamic Characteristics," Wind Energy, 12, pp. 752-767 (2009).

5. Leishman, J. G., "Dynamic Stall Experiments on the NACA 23012 Aerofoil," Experiments in Fluids, 9, pp. 49-58 (1990).

6. Ramsay, R. R., Hoffman, J., Gregorek, G. M., University, O. S. and Laboratory, N. R. E., "Effects of Grit Roughness and Pitch Oscillations on the S809 Airfoil," National Renewable Energy Laboratory, (1995).

7. Somers, D. M., Design and Experimental Results for the S809 Airfoil, National Renewable Energy Laboratory, Golden, Colorado (1997).

8. Sadeghi, H., Mani, M. and Ardakani, M. A., "Effect of Amplitude and Mean Angle of Attack on Wake of 
an Oscillating Airfoil," Proceedings of World Academy of Science: Engineering \& Technolog, 45, p. 125 (2008).

9. Wang, S., Ingham, D. B., Ma, L., Pourkashanian, M. and Tao, Z., "Numerical Investigations on Dynamic Stall of Low Reynolds Number Flow Around Oscillating Airfoils," Computers \& Fluids, 39, pp. 1529-1541 (2010).

10. Amiralaei, M. R., Alighanbari, H. and Hashemi, S. M., "An Investigation Into the Effects of Unsteady Parameters on the Aerodynamics of a Low Reynolds Number Pitching Airfoil," Journal of Fluids and Structures, 26, pp. 979-993 (2010).

11. Gharali, K. and Johnson, D. A., "Numerical Modeling of an S809 Airfoil Under Dynamic Stall, Erosion and High Reduced Frequencies," Applied Energy, 93, pp. 45-52 (2012).

12. Lu, K., Xie, Y. H., Zhang, D. and Lan, J. B., "Numerical Investigations Into the Asymmetric Effects on the Aerodynamic Response of a Pitching Airfoil," Journal of Fluids and Structures, 39, pp. 76-86 (2013).

13. Menter, F. R., "Two-Equation Eddy-Viscosity Turbulence Models for Engineering Applications," AIAA Journal, 32, pp. 1598-1605 (1994).

14. Wilcox, D. C., Turbulence Modeling for CFD, DCW industries, Inc., Lake Arrowhead, California (2006).
15. Karbasian, H. R., Esfahani, J. A. and Barati, E., "Effect of Acceleration on Dynamic Stall of Airfoil in Unsteady Operating Conditions," Wind Energy, DOI: 10.1002/we.1818 (2014).

16. Srinivasan, G. R., Ekaterinaris, J. A. and McCroskey, W. J., "Evaluation of Turbulence Models for Unsteady Flows of an Oscillating Airfoil," Computers \& Fluids, 24, pp. 833-861 (1995).

17. Streiner, S., Krämer, E., Eulitz, A. and Armbruster, P., "Aeroelastic Analysis of Wind Turbines Applying 3D CFD Computational Results," Journal of Physics: Conference Series, 75, p. 012015 (2007).

18. Moshizi, S. A., Nakhaei, M. H., Kermani, M. J. and Madadi, A., "Development of a Numerical Based Correlation for Performance Losses due to Surface Roughness in Axial Turbines," Journal of Mechanics, 30, pp. 631-642 (2014).

19. Moshizi, S. A., Madadi, A. and Kermani, M. J., "Comparison of Inviscid and Viscous Transonic Flow Field in VKI Gas Turbine Blade Cascade," Alexandria Engineering Journal, 53, pp. 275-280 (2014).

(Manuscript received January 15, 2015, accepted for publication June 5, 2015.) 